\title{
Subordinate-level categorization relies on high spatial frequencies to a greater degree than basic-level categorization
}

\author{
CHARLES A. COLLIN and PATRICIA A. MCMULLEN \\ Dalhousie University, Halifax, Nova Scotia, Canada
}

\begin{abstract}
In two experiments, category verification of images of common objects at subordinate, basic, and superordinate levels was performed after low-pass spatial filtering, high-pass spatial filtering, $50 \%$ phase randomization, or no image manipulation. Both experiments demonstrated the same pattern of results: Low-pass filtering selectively impaired subordinate-level category verification, while having little to no effect on basic-level category verification. Subordinate categorization consequently relies to a greater degree on high spatial frequencies of images. This vulnerability of subordinate-level processing was specific to a lack of high spatial frequency information, as opposed to other visual information, since neither high-pass filtering nor the addition of phase noise produced a comparable reduction in performance. These results are consistent with the notion that object recognition at basic levels relies on the general shapes of objects, whereas recognition at subordinate levels relies on finer visual details.
\end{abstract}

Objects can be identified at a number of levels of abstraction. For instance, a Collie dog can be correctly identified as an animal, a dog, or a collie. Rosch, Mervis, Gray, Johnson, and Boyes-Braem (1976) proposed that these identification terms lay within a taxonomy of levels from the superordinate (animal) to the subordinate (collie). Between these two extremes is the basic-level identifier $(d o g)$. Basic-level identification seems to display a number of processing advantages, relative to other levels. For instance, basic-level categories are verified most quickly (Johnson \& Mervis, 1997; Jolicœur, Gluck, \& Kosslyn, 1984; Murphy, 1991; Murphy \& Brownell, 1985; Murphy \& Smith, 1982; Rosch et al., 1976; Tanaka \& Taylor, 1991). Children learn basic-level names first (Horton \& Markman, 1980; Markman, 1989; Markman \& Hutchinson, 1984; Mervis \& Crisafi, 1982; Rosch et al., 1976). Adults use them most frequently in free-naming (Berlin, 1992; Rosch et al., 1976; Tanaka \& Taylor, 1991), and they are retained by patients with dementia of the Alzheimer's type even when superordinate verification is comparatively impaired (Purdy, McMullen, \& Freedman, 2001). Finally, a variety of image manipulations, including rotation (Hamm, 1998; Hamm \& McMullen, 1998), scrambling (Jaskie, 2001), and size reduction (Archambault, Gosselin, \& Schyns, 2000; Schyns \& Gosselin, 2002) affect basic-level categorization to a lesser degree than categorization at other levels does.

This work was supported by Grant NSERC 288346/2004 from the Natural Sciences and Engineering Research Council of Canada to C.A.C. and by Grant RG 0161/1999-B, P. McMullen PI from the Human Frontiers Science Program to P.A.M. Correspondence concerning this article should be addressed to C. A. Collin, School of Psychology, University of Ottawa, Ottawa, ON, K1N 6N5 Canada (e-mail: ccollin@uottawa.ca).
Although the superiority of the basic level is not without exceptions (Bailenson, Shum, Atran, Medin, \& Coley, 2002; Medin \& Atran, 2004; Mervis, 1987; Mervis \& Crisafi, 1982; Tanaka \& Taylor, 1991), it is well supported that the basic-level of identity generally has a processing advantage over other levels. However, there is some debate as to how this advantage comes about. Some have suggested that basic level identity is available first (Jolicœur et al., 1984), derived from either a holistic shape mechanism (e.g., Biederman 1987; Biederman \& Gerhardstein, 1993; Boucart \& Humphreys, 1992; Sanocki, 2001) or an analysis of distinctive basic features (Humphreys \& Riddoch, 1984; Jolicœur, 1990; McMullen \& Jolicœur, 1990, 1992). For instance, a dog can be recognized from either its general shape (a trunk with four legs, a tail, and a head) or its basic features (furriness, claws, and sharp teeth). These allow identification at the basic level but are nondistinguishing at the subordinate level. For instance, both beagles and collies have the same general shape and basic features. In order to perform a more precise identification at the subordinate level, further visual processing is required in order to extract fine visual details (Jolicœur et al., 1984).

Jolicœur et al. (1984) suggested that, following initial basic-level identification, superordinate and subordinate identification take place on the basis of different types of processing. Superordinate identification is done using further semantic analysis. For instance, once a dog is identified as such, further analysis on a semantic level determines whether it is part of the class animal. This is due to the fact that there are no perceptual features that reliably identify superordinate classes. Animals, for instance, have a wide variety of shapes and basic perceptual feature sets. Only semantics link them together into a larger class. On the other 
hand, identification at a subordinate level relies on further visual processing following basic-level identification. For instance, once a dog has been identified as such, further analysis of the fine details of its image leads to conclusions about whether or not it is a member of the class collie.

\section{Coarse-to-Fine Visual Analysis}

The concept of a time line of visual categorization working from general shape to fine details parallels a more general idea in visual perception that the visual system analyzes raw input in order from low to high spatial frequencies (Marr, 1982; Schyns \& Oliva, 1994) or from large global shapes to smaller local ones (Kimchi, 1992; Love, Rouder, \& Wisniewski, 1999; Navon, 1977; Sanocki, 2001). Low spatial frequencies are analyzed most quickly by the visual system (Aiken \& Loop, 1990; Breitmeyer, 1975; Schyns \& Oliva, 1994) and are, therefore, likely candidates for the information used in initial basiclevel identification of visual objects.

Although a simple, fixed, coarse-to-fine analysis of visual information does not seem adequate to explain all the data available (Amirkhiabani \& Lovegrove, 1996; Greaney \& MacRae, 1992; Kim, Ivry, \& Robertson, 1999; Lamb \& Robertson, 1989, 1990; Lamb \& Yund, 2000; Oliva \& Schyns, 1997; Schyns, 1998; Schyns \& Oliva, 1997; Shedden \& Reid, 2001; see Kimchi, 1992, for a review of earlier work), a general advantage of low spatial frequencies and global shapes remains well supported. In general, larger, global, low spatial frequency elements are processed preferentially relative to small, local, high spatial frequency elements (Kimchi, 1992), especially when the different levels are equated for conspicuity (Love et al., 1999).

\section{Visual Prototypes}

The idea that basic-level recognition involves processing general shape or basic features presupposes that all basic categories differ from one another in one or both of these criteria. But this is not always the case. For instance, cat and dog are basic semantic categories, but they have similar general shapes (a trunk, four legs, a head at one end, and a tail at the other) and basic features (furry, claws, and sharp teeth). So they appear to be part of the same perceptual category. On the other hand, the category lamp maps onto a wide variety of shapes and basic feature sets. So a single semantic category can contain numerous perceptual ones. From these examples, it is clear that there is an imperfect overlap between semantic and perceptual category structures. This is a potential source of confusion in interpreting studies on categorization.

Hamm (1998) has suggested that one can distinguish between semantic categories and what he calls visual prototypes, which are internal representations of classes of objects with the same general shape. In Hamm's model, the cognitive system has one visual prototype for both cat and $d o g$, despite the fact that they are different semantic categories. On the other hand, there are numerous visual prototypes that map to the semantic category lamp. Hamm suggests that in order to avoid difficulties in interpreta- tion, experiments should employ sets of distinct semantic categories that also map to distinct visual prototypes. For instance, the basic-level categories dog, bird, and bug differ markedly in shape, as well as in basic features, making them good choices.

\section{Categorization Tasks}

A number of tasks have been used to examine questions surrounding categorization (see, e.g., Rosch et al., 1976). In the experiments reported in the present article, we employed a verification task, in which a category name was presented first, followed by an object image. The subject was instructed to determine whether the object belonged to the category or not, and the specificity of the category name was varied from subordinate to superordinate across trials. This task has the advantage that it allows for a high degree of control over the level of identification of the image, as well as the order of processing. In a task in which the image is presented first, one cannot control whether subjects choose to initially analyze the image at a superordinate, a basic, or a subordinate level. Conversely, in a task in which a name and a picture are presented simultaneously, one cannot control whether subjects access the word first or the image first, adding noise to the data and difficulty to interpretation. In the experiments that follow, we were interested in determining the kinds of visual information needed to make category judgments at the different category levels. Because of this, we required maximum control over the level of categorization. The verification task afforded this.

The name-picture verification task also has the advantage that it equalizes chance-level performance between categorization levels. In a simple naming task, the probability of guessing the correct answer is lower for subordinatelevel than for basic-level identification, simply because there exist more subordinate-level categories. By making the task one in which the category is provided ahead of time and the subject must simply assess whether an object belongs to that category or not, we equalize chance-level performance at $50 \%$.

Although these advantages - strict control of categorization level and equalization of chance performancerecommend the name-picture verification task, it should be noted that it may not necessarily reflect the same cognitive process as some other categorization tasks. In a typical categorization task, a subject is given an image of an object and has to analyze the image to determine its identity at different levels of specificity, thus accessing a semantic category from a picture. In our task, the semantic category is given first, and the subjects are asked to subsequently determine whether a picture stimulus fits the label. This allows the subjects, upon seeing the label, to begin forming an anticipatory representation of the category of the object presented. Therefore, although the verification task has been used previously and has yielded results similar to those with more traditional categorization tasks (Hamm, 1998; Hamm \& McMullen, 1998), it might be the case that the two types of tasks are accessing some- 
what different aspects of categorization. The implications of this issue for our results are addressed in the General Discussion section.

\section{Summary and Hypotheses}

If global shape is generally extracted first and basic-level recognition is also generally achieved first, it seems plausible that the latter relies on the former. If this is the case, low spatial frequency information should be sufficient for basiclevel identification, but subordinate identification should rely on high spatial frequencies to a greater degree. This is because low frequencies carry information about the global shape of an object, but not about the local details of its texture, features, and so on (Love et al., 1999).

If low-pass filtering impairs subordinate categorization relative to basic, we may hypothesize that the information needed to make subordinate categorizations lies in the higher frequencies. However, it is also possible that subordinate recognition would suffer as a result of any sort of image degradation, not just those that remove high frequencies. Jolicœur et al. (1984) suggested that subordinate recognition requires more visual processing following entry-level identification at the basic level. If this is the case, any decrease in the visual information in an image might impair performance at subordinate recognition more than at basic. To support the idea that subordinate categorization relies on high frequencies per se, it is necessary to show that it is not impaired by image degradations that leave high-frequency information intact. With this in mind, we examined two other forms of image degradation besides low-pass filtering: (1) high-pass spatial filtering, which removes only low frequencies, and (2) $50 \%$ phase randomization, which degrades all frequencies equally. If subordinate-level recognition relies on high spatial frequencies, these two manipulations should not affect this level of categorization to a greater degree than the others.

In addition to testing subordinate- and basic-level categorization, we tested the effects of spatial filtering and phase noise on superordinate categorization. Jolicœur et al. (1984) have suggested that superordinate-level identification involves semantic processing following entry-level visual recognition at the basic level. If this is the case, we should expect to see similar effects of spatial frequency filtering and noise on superordinate- and basic-level identification.

In summary, the following experiments tested the effects of low-pass spatial filtering, high-pass spatial filtering, and $50 \%$ phase randomization on categorization at the subordinate, basic, and superordinate levels. We predicted that basic-level identification would be robust to all of these manipulations and that subordinate identification would be differentially vulnerable only to low-passing. Superordinate categorization was expected to follow the same pattern as basic-level categorization.

\section{EXPERIMENT 1}

In Experiment 1, the ability to confirm the congruency of names at different categorization levels (subordinate, basic, and superordinate) with grayscale images of objects under different spatial frequency conditions (low passed, high passed, and unfiltered) was tested. It was predicted that low-pass filtering would disproportionately impair subordinate-level categorization, as compared with basic or superordinate levels.

\section{Method}

Subjects. There were 27 subjects with a mean age of $19.6 \pm$ 5.8 years. All were students at Dalhousie University. All had normal or corrected-to-normal vision.

Materials. The experiment was presented using Matlab Version 5.2 for Macintosh (The Mathworks Inc., Natick, MA; www.mathworks. com) on a G3-300 computer. Matlab was also used to prepare stimuli, except where otherwise noted below. The monitor was a 17-in. Apple Studiodisplay CRT calibrated to provide a linear luminance profile.

The stimuli for the experiment consisted of 36 images of objects, along with 36 subordinate-level names, 6 basic-level names, and 2 superordinate-level names. The names are shown in Figure 1, which

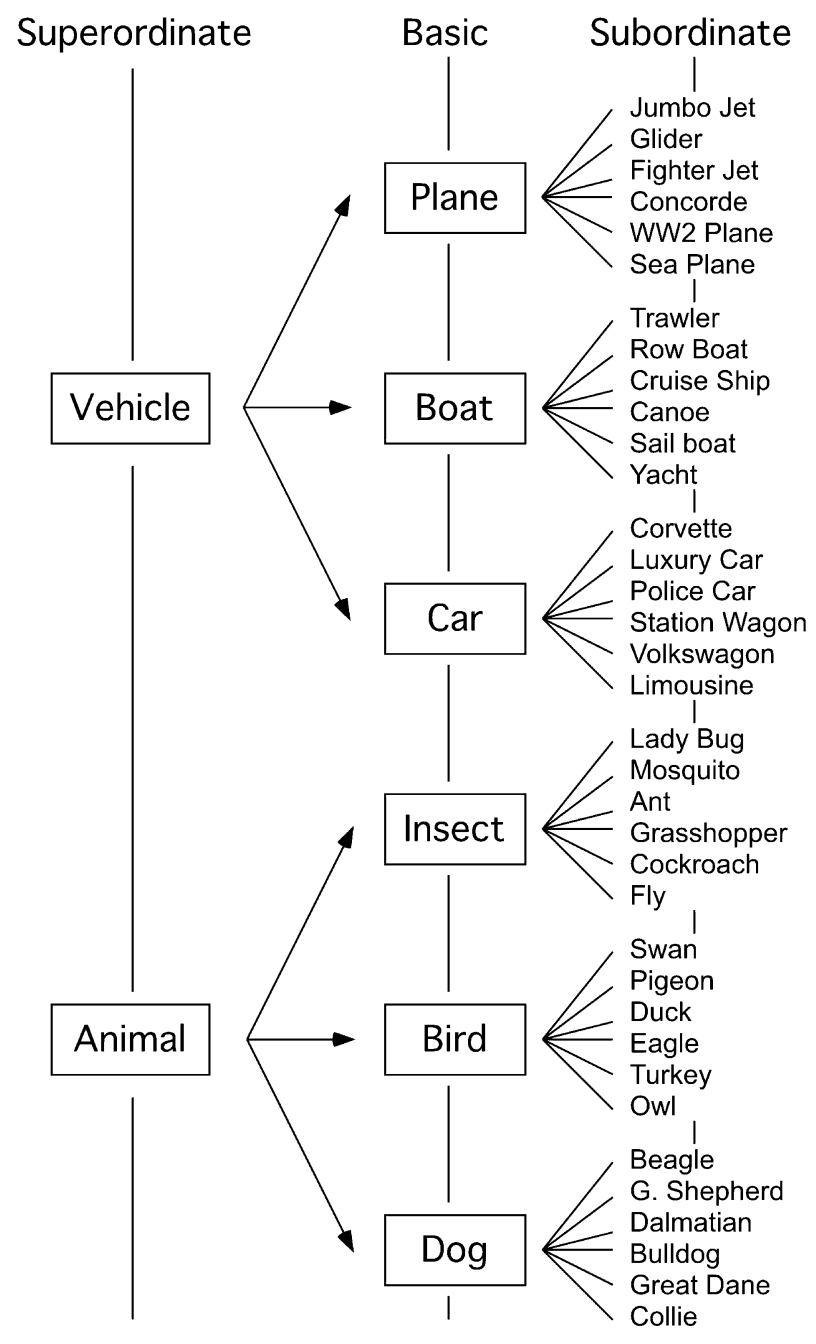

Figure 1. The name stimuli used in all the experiments. The figure shows the interelationships between the superordinate (left), basic (middle), and subordinate (right) stimuli. 
also shows their categorical relationships with one another. The superordinate- and basic-level names are the same as those used by Hamm (1998; Hamm \& McMullen, 1998). At the subordinate level, the names used were a subset of those used by Hamm (1998; Hamm \& McMullen, 1998). Hamm's stimulus set included 12 to 13 items from each of the basic-level categories. We selected 6 of his items from each basic category, all of which had received a mean typicality rating in his studies of at least 4.5 on a 7-point scale on which high typicality was assigned a value of 7 (Hamm, 1998, Experiments 1 and 5). It is important to note that Hamm used line drawings of the objects, whereas we used realistic grayscale images, so the ratings apply to our stimuli only to the extent that Hamm's subjects were evaluating the typicality of the subordinate object as a concept, rather than the individual image with which they were presented. It should also be noted that these categories were selected by Hamm on the basis of his recommendations that for studies in this area, categories should be chosen that are easily distinguished on the basis of both general shape and basic features, thus avoiding some difficulties in the interpretation of effects at the category level or the level of visual perception (Hamm, 1998; Hamm \& McMullen, 1998).

An example of our image stimuli is shown in Figure 2. The images were gathered from various Internet sources and were subsequently transformed to 8-bit grayscale format. The size of each object was scaled down so that the diagonal of its bounding box (excluding the background) was 140 pixels. This proved to be the best way to equate the sizes of the various objects, which had widely divergent aspect ratios. A diagonal of 140 pixels approximately corresponds to a height and width of 100 pixels in the case of an object with a 1:1 aspect ratio. The objects' backgrounds were removed using Adobe PhotoShop (www.adobe.com) and replaced with a $256 \times 256$ gray background of $38.4-\mathrm{cd} / \mathrm{m}^{2}$ luminance.
To produce the filtered images, the original object pictures were Fourier transformed into the frequency domain and then multiplied with a two-dimensional Butterworth filter of exponent 5 before being inverse Fourier transformed back into the spatial domain. The $50 \%$ cutoffs for the low-pass and high-pass Butterworth filters were 8 cycles/object diagonal and 16 cycles/object diagonal, respectively, where the object diagonal was 140 pixels. These translate into 5.7 cycles/object width and 11.3 cycles/object width, respectively, when an aspect ratio of 1:1 is assumed and, thus, a height and width of 100 pixels. The reason for choosing these cutoffs is addressed in the General Discussion section.

Procedure. Each subject underwent a practice task prior to the main task. The practice consisted of nine trials, one for each of the spatial frequency $\times$ category-level conditions. The stimuli used in the practice trials were pictures of vegetables and furniture, items from superordinate categories unrelated to those used in the main task. On each practice trial, the subject was asked to press a key to begin, after which one of the object names (e.g., furniture or beet) was presented for $3 \mathrm{sec}$. This was followed by an object picture that either matched the name or did not (e.g., a picture of a chair or a picture of a corn cob). The subject's task was to say, by means of yes and no keys on a keyboard, whether the name was congruent with the picture or not. The subject was instructed to answer as quickly as possible. Errors and reaction times (RTs) were gathered automatically.

The main task had a $3 \times 3$ fully factorial within-subjects design, with factors being spatial frequency filtering (low pass, high pass, or unfiltered) and categorization level (subordinate, basic, or superordinate). Trials were fully blocked across both factors, and the nine blocks were presented in random order. There were 72 trials per block, for a total of 648 trials. Half of the trials in each block were match trials, and half were mismatch trials. Mismatch pictures were

A)

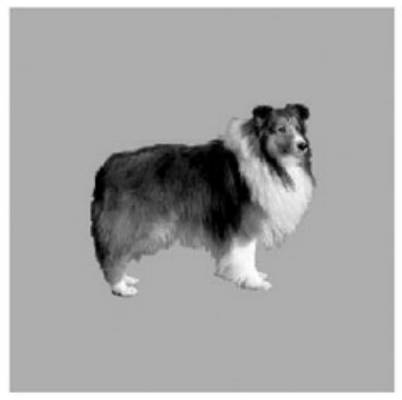

Unfiltered

B)

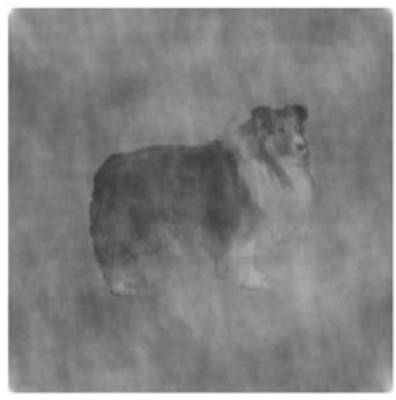

Unfiltered

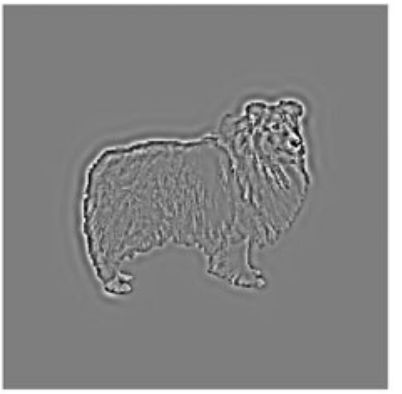

High-pass

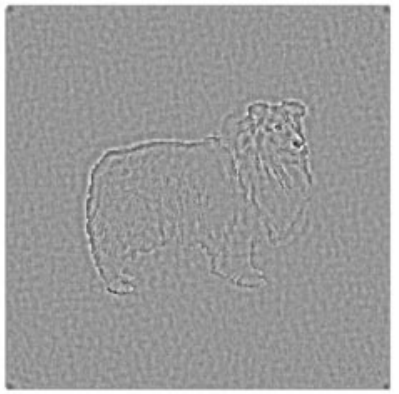

High-pass

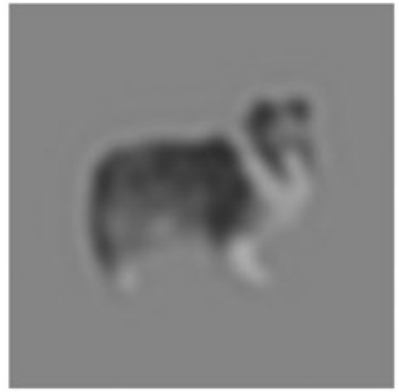

Low-pass

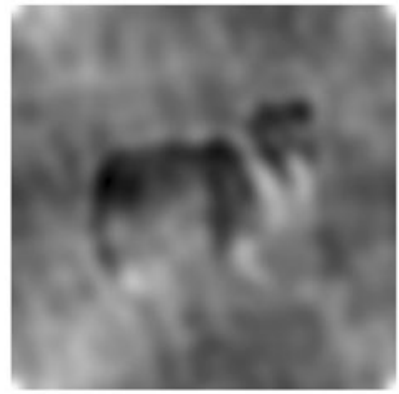

Low-pass

Figure 2. An example image stimulus as it appeared in the unfiltered, high-pass, and low-pass conditions. (A) The stimuli without phase noise, as used in Experiment 1. (B) The same stimuli with $50 \%$ phase noise added, as used in Experiment 2. 
always from the same category and level as the accompanying name. For instance, in the subordinate classification task, the name beagle would be followed by a picture of a different sort of dog, whereas in the basic-level task the distractor for the name $d o g$ would be a picture of a different sort of animal. The procedure on each trial was the same as that for the practice trials, as described above. Errors and RTs were gathered automatically.

\section{Results}

All the data reported for errors and RTs pertain to trials in which the word and the image matched. Only on match trials could it be certain that the images were identified at the level specified by the word. In contrast, mismatch trials could have involved processing at another level (for example, a collie may have been determined not to be a car because it is a dog, not because it is a collie).

Error rates. The mean error rate results in Experiment 1 are shown in Figure 3. Analyses of variance (ANOVAs) ${ }^{1}$ showed significant main effects of categorization $[F(2,52)=4.182, p=.0207]$ and spatial fre-
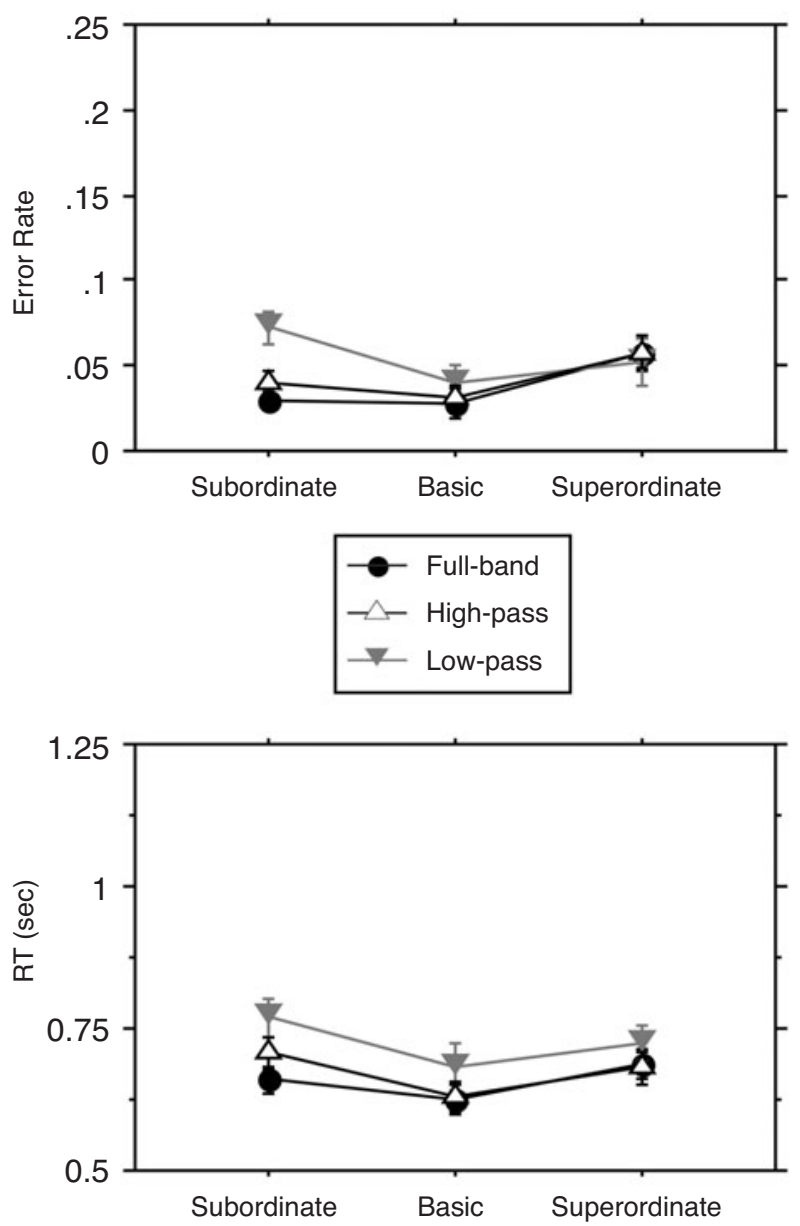

Figure 3. Results for Experiment 1. (A) Mean error rates and (B) mean reaction times (RTs) as a function of category level and spatial frequency filtering for match trials. Error bars represent $\pm 1 S E$. quency $[F(2,52)=4.603, p=.0144]$. There was also a significant interaction between these factors $[F(4,104)=$ $2.517, p=.0458]$.

An analysis of the simple main effects within each category level showed that the interaction was due to an effect of spatial frequency on errors for subordinate-level categorization $[F(2,114)=13.250, p<.0001]$, but not for superordinate-level $[F(2,114)=2.002, p=.1398]$ or basic-level $[F(2,114)=0.586, p=.558]$ categorization. A post hoc analysis (Fisher's PLSD, $\alpha=.05$ ) of the effect of spatial frequency within the subordinate categorization level showed that this was due to a significantly greater number of errors in the low-pass condition (7\%) than in either the high-pass $(3 \%)$ or the unfiltered $(3 \%)$ conditions. This result suggests that subordinate-level recognition is more vulnerable to removal of high spatial frequencies than basic and superordinate levels are.

A post hoc analysis (Fisher's PLSD, $\alpha=.05$ ) of the main effect of spatial frequency showed that this effect was due to an overall advantage of unfiltered images over low-passed ones ( $4 \%$ vs. $6 \%$, respectively). This effect was expected, since unfiltered images have more information than filtered ones. There was also a trend toward high-pass images having an advantage over low-pass ones $(p=.0535)$.

A post hoc analysis showed that the main effect of categorization was due to fewer errors with basic-level (3\%) than with superordinate-level $(6 \%)$ categorization overall. There was also a trend toward subordinate categorization producing more errors overall than basic categorization did $(p=.0758)$. These findings show an expected superiority of unfiltered images and basic-level categorization. The finding that superordinate-level errors were higher than subordinate-level errors was unexpected but was likely due to the use of photographic images in this study, rather than the line drawings that had been primarily used in previous work. Photographs provide more visual detail than line drawings. Because analysis of visual detail is needed for the process of subordinate-level identification (Jolicœur et al., 1984), photographs may produce superior performance at this level of categorization, relative to line drawings.

Reaction times. The RT data from Experiment 1 are shown in Figure 3, which shows mean RTs for correct trials only. An ANOVA showed significant main effects of categorization $[F(2,52)=4.182, p=.0207]$ and spatial frequency $[F(2,52)=4.603, p=.0144]$ on mean RT. There was no significant interaction. Analysis of median RTs showed substantively the same results.

Post hoc testing showed that the effect of categorization was due to an advantage for basic-level categorization (646 msec) over both subordinate-level $(712 \mathrm{msec})$ and superordinate-level (697 msec) categorization. This was expected on the basis of past research (Johnson \& Mervis, 1997; Jolicœur et al., 1984; Murphy, 1991; Murphy \& Brownell, 1985; Murphy \& Smith, 1982; Rosch et al., 1976; Tanaka \& Taylor, 1991). The effect of spatial frequency in this experiment was found to be due to an advantage for un- 
filtered images over both low-passed and high-passed images. This pattern was as expected, since unfiltered images provided more information with which to complete the task.

\section{Discussion}

The results of Experiment 1 suggest that subordinate recognition relies on high spatial frequencies to a greater degree than basic or superordinate categorization do. However, it is not clear from these results that it is the loss of high frequencies per se that is responsible for the effect. It is also possible that any image degradation might lead to relatively poorer subordinate-level performance, since this level is thought to involve the most visual analysis of the images (Jolicœur et al., 1984). Our finding that high-passing images (i.e., removing low spatial frequencies) does not impair subordinate recognition helps to narrow down the types of image degradation that can affect subordinate recognition, but it is of interest to further specify the types of information that impair or facilitate different levels of object categorization. To this end, we executed a methodology similar to that in Experiment 1 in Experiment 2 but added phase noise to the images. By comparing the results of Experiments 1 and 2, we should thus be able to determine the effects of this kind of image degradation as it interacts with spatial frequency filtering. In addition, this allows us to investigate whether the results of Experiment 1 will hold under more difficult visual conditions.

\section{EXPERIMENT 2}

Experiment 2 was similar to Experiment 1, except that we introduced $50 \%$ phase noise to the images. This kind of image degradation has the advantage that it produces noise that is in proportion to the energy at each spatial frequency level, unlike simple white noise, which has a different energy function than natural images. Indeed, using phase noise means that the noise will have exactly the same energy distribution as the image itself, because it consists only of frequency elements of the image that have been displaced. In this sense, it is analogous to a block scrambling of the image, but in the frequency domain instead of in the spatial domain.

In addition, adding noise was expected to increase errors, relative to the performance in Experiment 1. This was expected to correct for any potential ceiling effects in performance in the previous experiment.

\section{Method}

Subjects. There were 23 subjects with a mean age of $23.1 \pm$ 1.0 years. All were undergraduate students at Dalhousie University. All had normal or corrected-to-normal vision.

Materials. The materials and stimuli in Experiment 2 were similar to those in Experiment 1, except that the images of the objects had $50 \%$ phase noise added to them. That is, each image had noise added to its phase spectrum, so that only $50 \%$ of the original phase information remained. This was done after the images had been filtered. This had the effect of reducing the amount of useful information at all spatial frequency scales. Figure 2 shows an example of the stimuli used in this experiment.
Procedure. The design and procedure in Experiment 2 were the same as those in Experiment 1.

\section{Results}

As in Experiment 1, analyses of errors and RTs were restricted to the data from the match trials.

Error rates. The error rate results in Experiment 2 are shown in Figure 4. Overall, the error rates were much higher than those in the previous experiment (11.4\%). An ANOVA showed results similar to those in Experiment 1: significant main effects of both category level $[F(2,60)=$ $37.775, p<.0001]$ and spatial frequency $[F(2,60)=7.143$, $p=.0016]$, along with a significant interaction between these factors $[F(4,120)=2.925, p=.0238]$.

An analysis of the simple main effects within category levels showed that the interaction was due to the effects of spatial filtering within the basic and subordinate categorization levels, but not within the superordinate level. Post hoc testing (Fisher's PLSD, $\alpha=.05$ ) within these simple main effects showed that low-passed images pro-
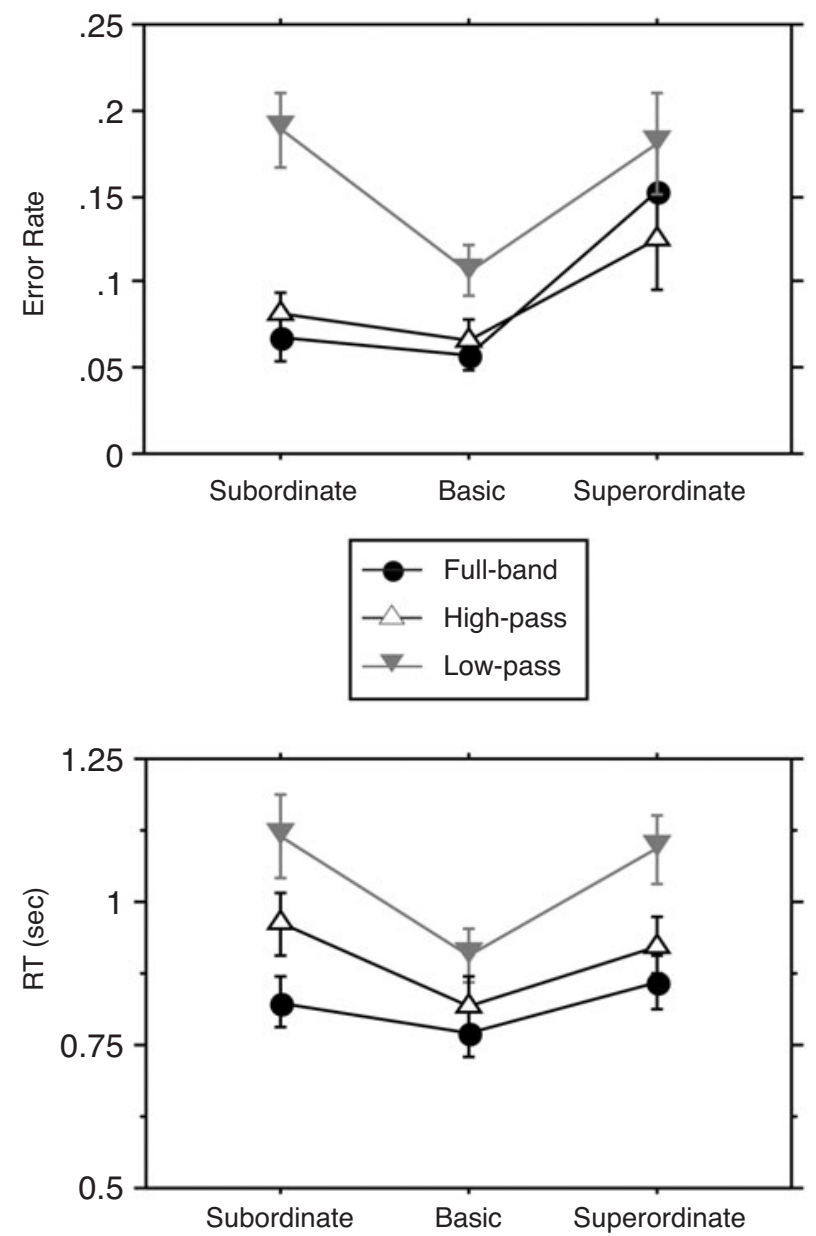

Figure 4. Results for Experiment 2. (A) Mean error rates and (B) mean reaction times (RTs) as a function of category level and spatial frequency filtering for match trials. Error bars represent $\pm 1 S E$. 
duced more errors $(19 \%)$ of subordinate categorization than either high-pass (8\%) or unfiltered (7\%) images did. Low-passed images also elicited more errors of basiclevel categorization (11\%) than either high-passed (7\%) or unfiltered $(6 \%)$ images did, although the effect at the subordinate level was about twice that at the basic level. This suggests that whereas basic-level categorization is affected by degradation of the images, subordinate-level recognition is more heavily influenced by removal of high frequencies. The fact that superordinate categorization was not affected in the same way as basic categorization was unexpected, and not in accord with the ideas of Jolicœur et al. (1984).

A post hoc analyses of the overall main effect of category level showed that this was due to an expected advantage of basic-level ( $8 \%$ errors) over superordinate-level $(15 \%)$ categorization. Post hoc analyses of the overall main effect of spatial frequency showed that this was due to more errors being committed overall with low-passed images ( $16 \%$ vs. $9 \%$ for both other image types), again an expected finding.

Reaction times. The RT results in Experiment 2 are shown in Figure 4. These are RTs for correct trials only. An ANOVA again showed results similar to those in Experiment 1: significant main effects of spatial frequency $[F(2,44)=52.156, p<.0001]$ and categorization level $[F(2,44)=16.180, p<.0001]$, but no interaction $[F(4,88)=2.205, p=.0750]$. As with Experiment 1 , an analysis of median RTs showed substantively the same results as an analysis of mean RTs.

Post hoc analysis showed that the effect of spatial frequency was due to all three levels being significantly different from one another: Unfiltered was fastest $(819 \mathrm{msec})$, followed by high-passed $(902 \mathrm{msec})$, followed by lowpassed $(1,038 \mathrm{msec})$. The effect of categorization level was due to an advantage for basic-level categorization $(832 \mathrm{msec})$ over both subordinate $(967 \mathrm{msec})$ and superordinate $(959 \mathrm{msec})$ levels.

Comparison of error rates from Experiments 1 and 2. To investigate the effects of phase noise on categorization, we compared the results of Experiments 1 and 2 , using a $2 \times 3 \times 3$ ANOVA with noise ( $0 \%$ vs. $50 \%$; i.e., Experiment 1 vs. Experiment 2), spatial frequency (low passed, high passed, or unfiltered), and categorization level (subordinate, basic, or superordinate) as factors. The design was mixed, with the noise factor being between subjects and the other two factors being within subjects. This analysis was performed for error rates only. Analysis across experiments is justified by the fact that the two experiments were identical in their methodology, except for the addition of phase noise in the second experiment. It was predicted that there would be a main effect of experiment and no interactions with this variable if the effects of category and spatial frequency were the same with or without noise.

An ANOVA revealed no significant three-way interaction $[F(4,192)=0.545, p=.7029]$. There was also no significant interaction of categorization level and noise level $[F(2,94)=2.216, p=.1148]$. This suggests that the effect of categorization level was the same regardless of noise level, indicating that subordinate categorization was not disproportionately impaired by the inclusion of noise under any of the spatial frequency conditions, including the unfiltered condition.

All the other effects and interactions were significant: spatial frequency $[F(2,96)=23.231, p<.0001]$, categorization level $[F(2,96)=10.650, p<.0001]$, noise $[F(1,48)=34.176, p<.0001]$, spatial frequency $\times$ categorization level $[F(4,192)=4.126, p=.0032]$, and spatial frequency $\times$ noise $[F(2,96)=8.901, p=.0003]$.

A post hoc analysis of the main effects showed that the effect of spatial frequency overall was due to the greater difficulty of the low-passed images, as compared with both high-passed and unfiltered images. The main effect of categorization level was a result of the usual advantage for basic-level categorization over both subordinate and superordinate categorization levels. The main effect of noise was due to noisy images being more difficult than those without noise. All of these main effects were expected results.

An analysis of the simple main effects of spatial frequency within each level of categorization showed that the interaction between these two factors was the same as that found in Experiments 1 and 2 individually. That is, lowpassed images produced more errors in both basic and subordinate categorization, with the effect on subordinate categorization being about twice as large.

An analysis of the simple main effects of spatial frequency within each level of noise showed that the interaction between these two factors was due to a slight difference in the pattern of errors: In Experiment 2 (with phase noise), low-passed images produced more errors than both unfiltered images and high-passed images did, whereas in Experiment 1 (no phase noise), the pattern was the same, except that the difference between low-passed and highpassed images did not quite reach significance $(p=.0535)$.

\section{Discussion}

As in Experiment 1, the results in Experiment 2 show that subordinate-level recognition is vulnerable to lowpassing. Unlike the previous experiment, this one showed an effect of low-passing on basic-level recognition as well, although this was much smaller than that on subordinatelevel recognition. This result was not surprising, since all levels of categorization will eventually suffer as images become sufficiently degraded. However, the fact that superordinate categorization was not affected in the same way as that of the basic level was unexpected. If superordinate categorization requires only semantic analysis following basic-level visual recognition (Jolicœur et al., 1984), we would expect that any image manipulations affecting the latter should affect the former.

The comparison of results from Experiments 1 and 2 showed that phase noise produced no differential deficit in subordinate categorization, although as was expected, it did produce more errors overall. This argues against the 
possibility that image degradations in general will disproportionately affect subordinate categorization. Taken together with those in the previous experiments, the data suggest that low spatial frequency information is sufficient for basic-level categorization, whereas subordinate categorization relies to a greater degree on higher spatial frequencies.

In summary, Experiment 2 replicated the results of Experiment 1, but with higher error rates, assuaging concerns about ceiling effects in the earlier experiment.

\section{GENERAL DISCUSSION}

Matching names to photographs of objects showed effects of spatial frequency filtering that depended on the category level of the name. With subordinate-level names (e.g., collie) a vulnerability to low-pass filtering was observed in both experiments. A similar, but smaller, effect was seen with basic-level names (e.g., dog) when the images were degraded with $50 \%$ phase noise in Experiment 2. No significant effects of spatial filtering were seen with superordinate names (e.g., animal). Although effect sizes in the first experiment were small, the relatively large effects seen in the second experiment, and the fact that the same pattern of results was found in both experiments, lend strong support to the conclusion that subordinate category verification relies to a greater degree on high spatial frequencies than does basic-level category verification. Overall, these results are compatible with the notion that basic-level categorization can rely on lower spatial frequencies, whereas subordinate categorization depends to a greater degree on high spatial frequencies. This in turn is compatible with the more general notion that image analysis usually proceeds from coarse to fine (Marr, 1982) or from global to local (Navon, 1977).

In interpreting our results relative to previous studies, it is important to consider the implications of the task used. In the present study, the subjects performed a task wherein they were presented with a category label first, followed by a picture. They were, therefore, potentially able to access an anticipatory representation of the object that they believed they were about to see. Assuming that they did so, their task was to determine whether the accessed representation matched the subsequently presented image. For low-passed images, there was disproportionate difficulty in doing this. Presumably, this was because a subordinatelevel representation contains distinguishing details not available in the low-passed image. These include such things as texture patterns (such as the distinctive color markings of collies and beagles) and metric part differences (such as the relative lengths of a bulldog's and a collie's legs). So, for instance, a subject might see the word collie, and access a representation of that subordinate class, including a collie's coloration and relatively long legs. When a low-passed image of the collie was presented, the subjects often erroneously said that it was not a collie, presumably because the distinguishing features in the representation were not perceptible in the image. On the other hand, high-passed and phase-scrambled images have their high spatial frequency information intact, making these detailed features available in the presented images. Therefore, there was little difficulty in matching the accessed subordinate-level representation to the presented image in the latter conditions.

In contrast to subordinate-level category verification, basic-level category verification was little impaired by low-passing. This result is compatible with the idea that basic-level representations contain an element of the general shapes of objects (Biederman, 1987; Hamm, 1998). Presumably, when a subject accessed a basic-level representation and attempted to match it to a low-passed image presented subsequently, a general shape match could be achieved. For example, when a subject saw the label $d o g$, they accessed a representation of that class, which included a visual prototype describing the general shape of all dogs. A similar general shape was perceptible in the low-passed image, and so a match could easily be assessed. Likewise, high-passed and phase-scrambled images retain the general shapes of the objects being presented, so those manipulations did not impair basic-level recognition.

As with basic-level categorization, superordinate category verification was not disproportionately impaired by low-passing. This result is compatible with Jolicœur et al.'s (1984) assertion that superordinate categories are accessed from perceptually based basic-level category representations. Presumably, subjects could not access a perceptual representation of the classes animal or vehicle, since there are no perceptual features that reliably define these categories, so they would, instead, have to wait to see the image, assess its basic-level category membership, and then perform further semantic analysis to determine whether it was a member of the previously presented superordinate category. Therefore, performance at this level was generally similar to that at the basic level.

An alternate explanation for the relative vulnerability of subordinate categorization to low-pass spatial filtering is that this level of identification is simply more vulnerable to any form of image degradation. However, we did not find a relative vulnerability of subordinate categorization to either high-pass spatial filtering or phase noise, even though these manipulations were effective in raising errors and RTs in general. High-passing an image removes only the low spatial frequencies, whereas phase noise scrambles the useful information at all frequencies equally. The finding that these image degradations did not disproportionately affect subordinate categorization strengthens our assertion that this task relies on high spatial frequencies, whereas coarser information is sufficient for basiclevel identification.

Our finding that low spatial frequencies are sufficient for basic-level categorization is in accordance with Schyns and colleagues' (Archambault et al., 2000; Schyns \& Gosselin, 2002) suggestion that the information needed for this level of categorization is more readily available than that required for subordinate categorization. In particular, 
Archambault et al. found that basic-level identification was unaffected by image size manipulations, whereas subordinate-level identification was impaired at small sizes. This finding could be due to size per se or to the loss of high spatial frequency information at small sizes. The fact that the present study showed a basic-level robustness to removal of high spatial frequencies while keeping image size constant demonstrates that spatial frequency was the critical manipulation in Archambault et al.'s experiment. Coarse-scale information is by its nature available at longer viewing distances and will typically be more robust to a variety of forms of degradation. This is because structural information at the object level becomes finer in spatial terms as the object moves farther away, gradually slipping below the acuity limit of the observer. Also, in natural images, low spatial frequencies have much higher contrast levels, with the energy dropping off as a $1 / f^{n}$ function, where $f$ is frequency and $n$ is an exponent between about 1.1 and 2.2 (see, e.g., Field \& Brady, 1997; Rainville \& Kingdom, 1999). This means that obscuration that reduces contrast - such as glare or fog - will tend to affect the higher frequencies more. Finally, low spatial frequencies are thought to be processed most quickly by the visual system (Aiken \& Loop, 1990; Breitmeyer, 1975; Schyns $\&$ Oliva, 1994), and this may partially explain the speed advantage seen in naming objects at the basic level. It is thus not surprising that the perceptual and cognitive systems might be set up to rapidly extract the invariant basiclevel identity of an object from low spatial frequency information. This level of identity is likely sufficient to allow simple adaptive responses, such as avoidance and approach, and would thus allow fast responses to sudden appearances of objects in the environment.

In discussing the effects of spatial frequency on recognition, an important consideration is the operational definition of low versus high spatial frequency bands. Typically, these are defined rather arbitrarily. In the present experiments, the choice of the spatial cutoffs was based on previous work showing that removing spatial frequency information above 16 cycles/object or below 8 cycles/object impairs both face and object recognition, likely because only information within this band contains sufficiently high levels of both contrast and structural information to aid recognition (Boutet, Collin, \& Faubert, 2003). Therefore, it was expected that spatially filtering images at these cutoffs would produce significant impairment of recognition overall, an expectation borne out by the results.

Another important consideration in discussing spatial frequency effects on recognition is that the scale of information useful for identifying different classes of objects varies (e.g., Vannucci, Viggiano, \& Argenti, 2001). Therefore, there will almost certainly be some variability in the difficulty of recognizing different object classes when their images are filtered at any given cutoff. However, there do exist striking statistical regularities in the distribution of energy and information across spatial frequency in natural images overall (see, e.g., Field \& Brady, 1997; Rainville \& Kingdom, 1999), so it is unlikely that object recognition performance, relative to spatial filtering, will be completely idiosyncratic. That is, it seems likely that our results have some degree of generalizability to other object classes.

One implication of the present study's findings is that object recognition researchers should be careful to consider the category level at which objects are being identified in their studies. Tjan and Legge (1998) pointed out that changes in the objective visual similarity of object stimuli can lead to very different conclusions about how recognition works. Schyns (1998) points out that a consideration of both task requirements and available visual information is necessary for an understanding of object recognition and categorization. In a related way, we suggest that there will be different information needs for tasks requiring different levels of categorization. Free-naming will generally elicit basic-level identification, whereas word-picture matching could require any level from superordinate to subordinate. Our results suggest that different levels of spatial frequency information are necessary for these tasks.

An aspect of our data that bears examination is the fact that subordinate categorization was generally about as difficult as basic-level for unfiltered and high-passed images. This pattern is unlike that found in past research, which has consistently shown that subordinate categorization is more difficult. One possible explanation for this discrepancy is that whereas in much of the past work on categorization line drawings have been used as stimuli (e.g., Hamm \& McMullen, 1998; Jolicœur et al., 1984; Rosch et al., 1976), we used realistic grayscale images. These present a richer amount of visual information and may aid in the detailed visual analysis needed for subordinate categorization. Indeed, face recognition, a highly subordinate categorization task, shows a disproportionate vulnerability to the reduction of images to line drawings (Bruce, Hanna, Dench, Healy, \& Burton, 1992; Leder, 1996).

In summary, our findings show that low spatial frequencies are sufficient for basic-level category verification and that subordinate category verification relies to a greater degree on high spatial frequencies. This suggests that the robustness of basic-level identification arises at least partially from the easier availability and higher energy of coarse scale information in natural images. One limitation of this study is that we examined only single levels of our spatial frequency manipulations. Our statements about the relative reliance of different levels of categorization on different spatial ranges must, therefore, remain qualitative. Further studies should be able to provide a set of spatial frequency thresholds separating the ranges needed for the different categorization tasks.

\section{REFERENCES}

Aiken, B. E., \& Loop, M. S. (1990). Visual reaction time of cats to different spatial frequencies. Neuroscience, 5, 557-564.

Amirkhiabani, G., \& Lovegrove, W. J. (1996). Role of eccentricity and size in the global precedence effect. Journal of Experimental Psychology: Human Perception \& Performance, 22, 1434-1447. 
Archambault, A., Gosselin, F., \& Schyns, P. G. (2000). A natural bias for the basic-level? In L. R. Gleitman \& A. K. Joshi (Eds.), Proceedings of the Twenty-Second Annual Conference of the Cognitive Science Society. NJ: Erlbaum. Available at www.cognitivesciencesociety.org/cogsci.html.

Bailenson, J. N., Shum, M., Atran, S., Medin, D. L., \& Coley, J. (2002). A bird's eye view: Biological categorization and reasoning within and across cultures. Cognition. 84, 1-53.

BERLIN, B. (1992). Ethnobiological classification: Principles of categorization of plants and animals in traditional societies. Princeton, NJ: Princeton University Press.

Biederman, I. (1987). Recognition-by-components: A theory of human image understanding. Psychological Review, 94, 115-147.

Biederman, I., \& Gerhardstein, P. C. (1993). Recognizing depthrotated objects: Evidence and conditions for three-dimensional viewpoint invariance. Journal of Experimental Psychology: Human Perception \& Perception, 18, 121-133.

Boucart, M., \& Humphreys, G. W. (1992). Global shape cannot be attended without object identification. Journal of Experimental Psychology: Human Perception \& Performance, 18, 785-806.

Boutet, I., Collin, C. A., \& Faubert, J. (2003). Configural face encoding and spatial frequency information. Perception \& Psychophysics, 65, 1078-1093.

BREITMEYER, B. G. (1975). Simple reaction time as a measure of the temporal response properties of transient and sustained channels. $\mathrm{Vi}$ sion Research, 15, 1411-1412.

Bruce, V., HanNa, E., Dench, N., Healy, P., \& Burton, A. M. (1992). The importance of "mass" in line drawings of faces. Applied Cognitive Psychology, 6, 619-628.

FIELD, D. J., \& BRADY, N. (1997). Visual sensitivity, blur and the sources of variability in the amplitude spectra of natural scenes. Vision Research, 37, 3367-3383.

Greaney, J., \& MacRAE, A. W. (1992). The order of visual processing: Top-down, bottom-up, middle-out, or none of these? Bulletin of the Psychonomic Society, 30, 255-257.

HAMM, J. (1998). Object orientation and levels of identity. Dissertation Abstracts International, 59, 893B.

HAMM, J., \& MCMulLEN, P. (1998). Effects of orientation on the identification of rotated objects depend on the level of identity. Journal of Experimental Psychology: Human Perception \& Performance, $\mathbf{2 4}_{2}$ 413-426.

HorTOn, M. S., \& Markman, E. M. (1980). Developmental differences in the acquisition of basic and superordinate categories. Child Development, 51, 708-719.

HumphreYs, G. W., \& RIDDOCH, M. J. (1984). Routes to object constancy: Implications from neurological impairments of object constancy. Quarterly Journal of Experimental Psychology, 36A, 385-415.

JASKIE, K. E. (2001). Mechanisms of orientation-invariant identification of objects. Unpublished master's thesis, Dalhousie University, Halifax.

Johnson, K. E., \& Mervis, C. B. (1997). Effects of varying levels of expertise on the basic level of categorization. Journal of Experimental Psychology: General, 126, 248-277.

JoLICEUR, P. (1990). On the role of mental rotation and feature extraction in the identification of disoriented objects: A dual-systems theory. Mind \& Language, 5, 387-410.

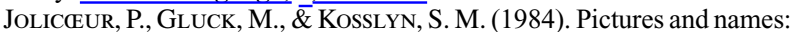 Making the connection. Cognitive Psychology, 19, 31-53.

KIM, N., IVRY, R. B., \& RobERTSON, L. C. (1999). Sequential priming in hierarchically organized figures: Effect of target level and target resolution. Journal of Experimental Psychology: Human Perception \& Performance, 25, 715-729.

KIMCHI, R. (1992). Primacy of wholistic processing and global/local paradigm: A critical review. Psychological Bulletin, 112, 24-38.

LAmb, M. R., \& Robertson, L. C. (1989). Do response time advantage and interference reflect the order of processing of global- and locallevel information? Perception \& Psychophysics, 46, 254-258.

LAMB, M. R., \& RoBERTSON, L. C. (1990). The effect of visual angle on global and local reaction times depends on the set of visual angles presented. Perception \& Psychophysics, 47, 489-496.
LAMB, M. R., \& YUnd, E. W. (2000). The role of spatial frequency in cued shifts of attention between global and local forms. Perception \& Psychophysics, 62, 753-761.

LEDER, H. (1996). Line drawings of faces reduce configural processing. Perception, 25, 355-366.

Love, B. C., Rouder, J. N., \& Wisniewski, E. J. (1999). A structural account of global and local processing. Cognitive Psychology, $\underline{\mathbf{3 8}}$, 291-316.

MARKMAN, E. M. (1989). Categorization and naming in children: Problems of induction. Cambridge, MA: MIT Press.

Markman, E. M., \& Hutchinson, J. E. (1984). Children's sensitivity to constraints on word meanings: Taxonomic vs. thematic relations. Cognitive Psychology, 16, 1-27.

MARR, D. (1982). Vision: A computational investigation into the human representation and processing of visual information. San Francisco: Freeman.

McMullen, P. A., \& Jolicceur, P. (1990). The spatial frame of reference in object naming and discrimination of left-right reflections. Memory \& Cognition, 18, 99-115.

MCMullen, P. A., \& JoLICEUR, P. (1992). The reference frame and the effects of orientation on finding the tops of rotated objects. Journal of Experimental Psychology: Human Perception \& Performance, $\underline{\mathbf{1 8}}_{2}$ 807-820.

MEDIN, D. L., \& ATRAN. S. (2004). The native mind: Biological categorization and reasoning in development across cultures. Psychological Review, 111, 960-983.

MERVIS, C. B. (1987). Child-basic object categories and early lexical development. In Neisser (Ed.), Concepts and conceptual development: Ecological and intellectual factors in categorization (Emory Symposia in Cognition, Vol. 1, pp. 201-233). New York: Cambridge University Press.

Mervis, C. B., \& Crisafi, M. A. (1982). Order of acquisition of subordinate-, basic-, and superordinate-level categories. Child Development, 53, 258-266.

MURPHY, G. L. (1991). Parts in object concepts: Experiments with artificial categories. Memory \& Cognition, 19, 423-438.

Murphy, G. L., \& BROWNELL, H. H. (1985). Category differentiation in object recognition: Typicality constraints on the basic category advantage. Journal of Experimental Psychology: Learning, Memory, \& Cognition, 11, 70-84.

MURPHY, G. L., \& SMITH, E. E. (1982). Basic level superiority in picture categorization. Journal of Verbal Learning \& Verbal Behavior, 21, 1-20.

NAVON, D. (1977). Forest before trees: The precedence of global features in visual perception. Cognitive Psychology, 9, 353-383.

Oliva, A., \& Schyns, P. G. (1997). Coarse blobs or fine edges? Evidence that information diagnosticity changes the perception of complex visual stimuli. Cognitive Psychology, 34, 72-107.

Purdy, K. S., McMullen, P. A., \& Freedman, M. (2001, April). Changes to the object recognition system in patients with dementia of the Alzheimer's type. Paper presented at the Annual Baycrest/Rotman Institute Conference on Dementia, Toronto.

Rainville, S. J. M., \& Kingdom, F. A. A. (1999). Spatial-scale contribution to the detection of mirror symmetry in fractal noise. Journal of the Optical Society of America A, 16, 2112-2123.

Rosch, E., Mervis, C. B., Gray, W. D., Johnson, D. M., \& BoyesBraem, P. (1976). Basic objects in natural categories. Cognitive Psychology, 8, 382-452.

SANOCKI, T. (2001). Interaction of scale and time during object identification. Journal of Experimental Psychology: Human Perception \& Performance, 27, 290-302.

SchYNS, P. G. (1998). Diagnostic recognition: Task constraints, object information, and their interactions. Cognition, 67, 147-179.

Schyns, P. G., \& Gosselin, F. (2002, May). A natural bias for basiclevel object categorizations. Paper presented at the Annual Meeting of the Vision Sciences Society, Sarasota, FL.

Schyns, P. G., \& Oliva, A. (1994). From blobs to boundary edges: Evidence for time- and spatial-scale-dependent scene recognition. Psychological Science, 5, 195-200.

SchYNS, P. G., \& OLIVA- A. (1997). Flexible, diagnosticity-driven, rather 
than fixed, perceptually determined scale selection in scene and face recognition. Perception, 26, 1027-1038.

SHEDDEN, J. M., \& REID, G. S. (2001). A variable masking task produces symmetrical interference between global information and local information. Perception \& Psychophysics, 63, 241-252.

TANAKA, J. W., \& TAYLOR, M. (1991). Object categories and expertise: Is the basic level in the eye of the beholder? Cognitive Psychology, $\underline{\mathbf{2 3}}_{2}$ 457-482.

TJAN, B. S., \& LegGe, G. E. (1998). The viewpoint complexity of an object-recognition task. Vision Research, 38, 2335-2350.

VanNucCi, M., Viggiano, M. P., \& ARGENTI, F. (2001). Identification of spatially filtered stimuli as function of the semantic category. $\operatorname{Cog}$ nitive Brain Research, 12, 475-478.

\section{NOTE}

1. All results were analyzed with both parametric statistics (ANOVA on error rates) and nonparametric statistics (Friedman's $F$ on $A^{\prime}$ scores). These showed substantively the same results.

(Manuscript received January 12, 2004; accepted for publication June 7, 2004.) 